\title{
Photon excitation of the third molecular continuum in solid krypton
}

\author{
A. N. Ogurtsov and E. V. Savchenko \\ B. Verkin Institute for Low Temperature Physics and Engineering, \\ National Academy of Sciences of Ukraine, Lenin Ave., 47, 310164, Kharkov, Ukraine
}

J. Becker, M. Runne, and G. Zimmerer

II. Institut für Experimental Physik der Universität Hamburg, 22761 Hamburg, Germany

Submitted May 26, 1997, revised June 6, 1997

\begin{abstract}
Luminescence study of the near-ultraviolet continuum in nominally pure solid krypton under selective excitation by synchrotron radiation is presented. Intrinsic nature of the emission has been established. Clear threshold behavior at the band gap energy reveals the key role of the electron-hole pairs in a population of the continuum forming states.
\end{abstract}

PACS: 78.55.Hx, 82.50.Fv

In addition to the well-known vacuum ultraviolet (VUV) continua stemming from the decay of self-trapped excitons, wide near-UV luminescence bands were observed in rare gas solids [1]. A rebirth of interest in their origin has been stimulated by a prospect of the development of excimer lasers operating in a near-UV range of spectra [2]. Despite the active current theoretical [3] and experimental studies in gas [4-7], condensed phases [8-10], and cluster beams [11], the origin of these bands (the so-called third continua) is still under discussion.

Synchrotron radiation is a very valuable tool for elucidation of primary processes of emitting state population. Luminescence study of the third continuum following selective excitation with synchrotron radiation was performed on solid Xe (Ref. 8) and solid Ar (Ref. 10). In the present paper, the results of the first analysis of the near-UV luminescence of solid krypton following primary selective excitation with synchrotron radiation in the excitonic range and the range of band-to-band excitation are presented [12].

The experiments were performed at the SUPERLUMI experimental station of HASYLAB at DESY [13] in an ultrahigh vacuum environment (a basic pressure in the experimental chamber did not exceed the $\left.10^{-10} \mathrm{mbar}\right)$. The samples were grown from a $\mathrm{Kr}$ gas $(99.99 \%)$ in a special cell attached to a helium cryostat. Preparation of samples under isobaric $(P=80 \mathrm{~Pa})$ conditions with a constant rate of cooling $0.1 \mathrm{~K} / \mathrm{s}$ in the temperature range 80 $60 \mathrm{~K}$ results in high transmittance samples with a small quantity of initial defects of structure. The samples contained inevitable amount of Xe impurity. The Xe concentration is estimated to be in a low ppm range [14]. After condensation the cell was opened and the measurements of photoluminescence and excitation spectra were performed. Selective photon excitation was carried out with $\Delta \lambda=2.5 \AA$. In the luminescence and the excitation spectra, the luminescence was spectrally dispersed with a $0.5-\mathrm{m}$ Pouey monochromator equipped with a solar-blind photomultiplier $(\Delta \lambda=15 \AA)$.

The luminescence spectrum in the region of selftrapped exciton emission (the $M$-band at $8.6 \mathrm{eV}$ ) and the third continuum at $5.3 \mathrm{eV}$ is shown in Fig. 1. The $M$-band consists of four wellknown $[15,16] M$-subbands originating from the radiative decay of $\mathrm{Kr}_{2}^{*}\left(M_{1}, M_{2}\right)$ and $\mathrm{XeKr}^{*}$ $\left(M_{1}^{\prime}, M_{2}^{\prime}\right)$ molecular centers, which are self-trapped in the regular lattice and trapped at the initial and electronically induced defects of the structure. A narrow band $A$ originats from $\mathrm{Xe}$ atoms in the $\mathrm{Kr}$ matrix [17]

At low temperatures the third continuum reveals two components: a $H$-band at $5.3 \mathrm{eV}$ with FWHM $=0.3 \mathrm{eV}$ and a $H^{\prime}$-subband at $4.9 \mathrm{eV}$ with FWHM $=0.4 \mathrm{eV}$. The $H^{\prime}$-subband disappears on heating of the sample, whereas the $H$-band only reduces its intensity by half, beginning with 


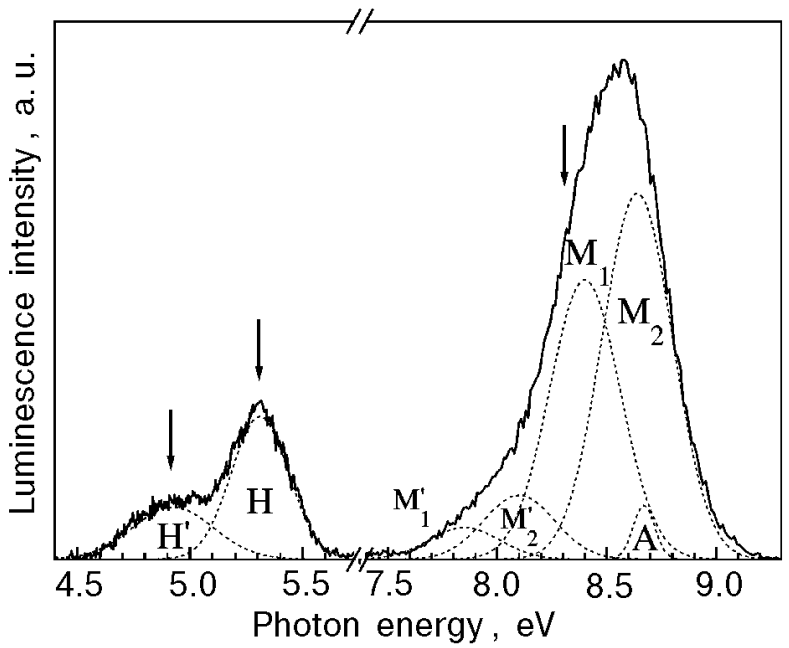

Fig. 1. The liminescence spectrum of solid krypron excited by $h v=14.2 \mathrm{eV}$ at $T=5 \mathrm{~K}$. The arrows indicate the photon energies at which the excitation spectra in Fig. 2 were measured.

$T=35 \mathrm{~K}$. The main component which peakes at $5.3 \mathrm{eV}$ and which remains in the luminescence spectrum, is thought to be of intrinsic nature.

In order to gain insight into the origin of this band, we measured the excitation spectrum. Figure 2 shows the excitation spectra of the $H^{-}$, the $H^{\prime}$-, and the $M$-bands recorded at the luminescence photon energies indicated in Fig. 1 by arrows. The choice of emission energy $(8.3 \mathrm{eV})$ for measuring the excitation spectrum of the $M$-band is dictated by its complex internal structure due to the presence of small quantity of Xe impurity. Note that at the chosen energy the contribution of the the $M_{1}$ subband is dominant. The excitation spectrum of the $M_{1}$ subband shows the well-known fine structure in

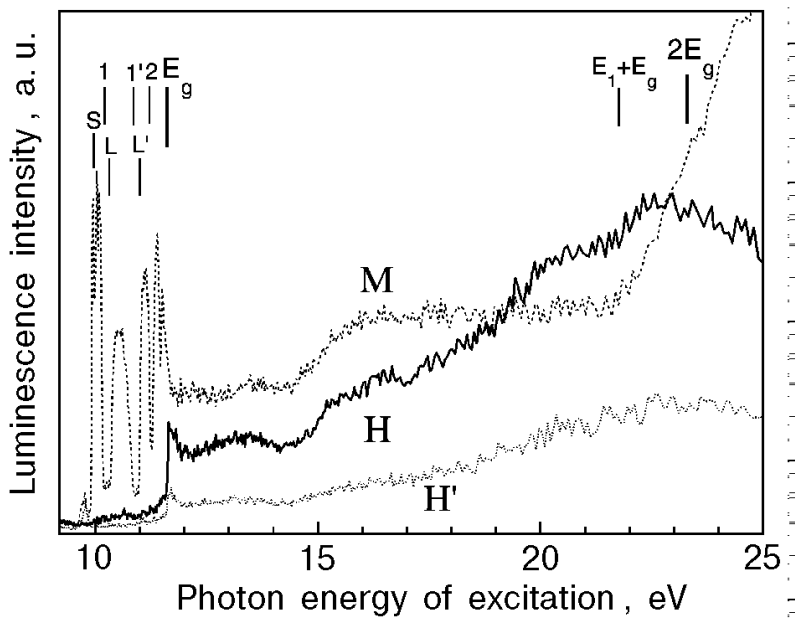

Fig. 2. The excitation spectra of $H-, H^{\prime}-$, and $M$-bands measured at luminescence photon energies indicated in Fig. 1 by arrows. The positions of bulk transverse $\left(1,1^{\prime}, 2\right)$, bulk longitudinal $\left(L, L^{\prime}\right)$, and surface $(S)$ excitons are marked at the top of the curves according to Ref. 1. the excitonic range of the spectrum [18]. The most prominent features were observed in the region below the first member of the bulk excitons $\Gamma(3 / 2)$. This fact clearly demonstrates the association of the subband with the structure defects and with the surface region of the sample. In addition, the excitation specrum of the $M_{1}$ subband yields a step-like behavior at the energy $E=21.78 \mathrm{eV}$, which exceeds the band gap energy $E_{g}=11.61 \mathrm{eV}$ by the lowest exciton energy $E_{1}=10.17 \mathrm{eV}$. The energy $E_{1}+E_{g}$ determines the threshold for creation of excitons during inelastic scattering of secondary photoelectrons by valence electrons [19].

To check the probable origin of the $H$-band from impurities, we measured the excitation spectrum in a transparency range of the $\mathrm{Kr}$ host. We found that this band cannot be excited below the range of intrinsic absorption. Moreover, the band in question cannot be excited in the excitonic range of energies $\left(E_{1}=E<E_{q}\right)$ and its behavior is in contrast with that of the extrinsic $M_{1}$ subband. The tests confirm beyond any doubt the intrinsic nature of the $H$-band in nominally pure solid krypton. The excitation spectrum of the $H$-band yields a pronounced threshold at the band gap energy $E_{g}$ and a maximum at $2 E_{g}$. The clear threshold behavior testifies that the creation of free electron-hole pairs is the primary process of population of continuum forming states. If the energy position of the $H$-band is taken into account, the direct recombination of free electrons and holes should be excluded. It is well known that free holes are rapidly self-trapped in atomic cryocrystals [20], forming molecular-ionlike centers. The radiative recombination of selftrapped holes with electrons results in the $M$-band emission in the VUV range of the spectra and is excluded as a probable channel of population of the third continuum forming states. A more likely assumption about the origin of the $H$-band seems to be the radiative transition from the excited states of the krypron molecular ion to lower-lying repulsive states. According to this assumption, the population of the continuum forming states is a two-step process: self-trapping of holes followed by their excitation. The key role of the self-trapped holes in the formation of near-UV emission from nominally pure solid krypton is evident from the observed features of the excitation spectrum and from the temperature behavior of the $H$-band - quenching with increasing temperature starting at $35 \mathrm{~K}$, where electrons are released from their traps [21]. The nature of the $H^{\prime}$-band is presently not clear. Perhaps it originates from heteronuclear krypton-xenon ionic centers. 
Detailed assignement of the emitting states requires further investigation.

Financial support by the BMFT (grant 05650 GUB) and under the PECO-Project is gratefully acknowledged.

1. I. Ya. Fugol', Adv. Phys. 1, 1 (1978).

2. H. Langhoff, Opt. Commun. 68, 31 (1988).

3. C. Cachoncinlle, J. M. Pouvesle, G. Durand, and F. Spiegelmann, J. Chem. Phys. 96, 6085 (1992).

4. W. Krotz, A. Ulrich, B. Bush, G. Ribitzki, and J. Wieser, Phys. Rev. A43, 6089 (1991).

5. A. Kh. Amirov, O. V. Korshunov, and V. F. Chinnov, $J$. Phys. B: At. Mol. Opt. Phys. 27, 1753 (1994).

6. H. Langhoff, J. Phys. B: At. Mol. Opt. Phys. 2, L709 (1994).

7. D. E. Johnson, Chem. Phys. Lett. 238, 71 (1995).

8. M. Hävecker, M. Runne, and G. Zimmerer, J. Electron Spectrosc. Rel. Phenom. 79, 103 (1996).

9. O. N. Grigorashchenko, S. A. Gubin, A. N. Ogurtsov, and E. V. Savchenko, J. Electron Spectrosc. Rel. Phenom. 79, 107 (1996).

10. A. Ogurtsov, M. Runne, A. Karl, J. Becker, and G. Zimmerer, HASYLAB Annual Report 1995, DESY, Hamburg (1996), p. II-75.

11. E. Rühl, C. Heinzel, and H. W. Jochims, Chem. Phys. Lett. 211, 403 (1993).
12. J. Becker, A. N. Ogurtsov, M. Runne, E. V. Savchenko, and G. Zimmerer, HASYLAB Annual Report 1996, DESY, Hamburg (1997), p. 247.

13. G. Zimmerer, Nucl. Instr. Meth. Phys. Res. A308, 178 (1991)

14. D. Varding, J. Becker, L. Frankenstein, B. Peters, M. Runne, A. Schröder, and G. Zimmerer, Low Temp. Phys. 19, 427 (1993).

15. E. V. Savchenko, A. N. Ogurtsov, O. N. Grigorashchenko, and S. A. Gubin, Chem. Phys. 189, 415 (1994).

16. A. N. Ogurtsov, E. V. Savchenko, S. A. Gubin, O. N. Grigorashchenko, M. Runne, A. Karl, J. Becker, and G. Zimmerer, in: Excitonic Processes in Condensed Matter, M. Schreiber (ed.), Dresden University Press, Dresden (1996).

17. W. Laasch, PhD Thesis, The University of Hamburg (1992); Internal Rep. DESY-HASYLAB 92-07, December 1992.

18. Ch. Ackermann, R. Brodmann, U. Hahn, A. Suzuki, and G. Zimmerer, Phys. Status. Solidi B74, 579 (1976).

19. H. Möller, R. Brodmann, G. Zimmerer, and U. Hahn, Solid State Commun. 20, 401 (1976).

20. K. S. Song and R. T. Williams, Self-Trapped Excitons, Springer Series in Solid-State Sciences 105, Springer-Verlag Berlin, Heidelberg, New York (1993).

21. M. Kirm and H. Niedrais, J. Luminesc. 60-61, 611 (1994). 